\section{Fehlende Antikörper bedingen Schwere der fetalen Parvovirus-Infektion}

\author{
Parvovirus B19 kann diaplazentar von der Mutter auf den Feten übertragen \\ werden. Abhängig vom Gestationszeitpunkt kommt es zum materno-fetalen \\ Transfer von spezifischen IgG, was zur Virusneutralisation führt und den \\ klinischen Verlauf der fetalen Infektion positiv beeinflusst. Sicher feststellen \\ lässt sich eine fetale B19V-Infektion nur durch den Nachweis von \\ viraler B19V-DNA.
}

$\mathrm{D}_{\mathrm{in}}^{\mathrm{ie}}$ ie humane Parvovirus B19 (B19V)Infektion kann in der Schwangerschaft einen Hydrops fetalis mit resultierendem intrauterinen Fruchttod verursachen. Wie seit Längerem bekannt, hat das Virus einen ausgeprägten Tropismus zu fetalen erythroiden Vorläuferzellen, in denen es sich lytisch vermehrt. Nach der maternalen Infektion kommt es in 25-33\% zu einer diaplazentaren Infektion. Das Ausmaß der fetalen Infektion ist sehr unterschiedlich: von asymptomatisch bis zu schwersten hydropischen Veränderungen. Bei der Pathogenese des Hydrops ist die Infektion der erythropoetischen Zellen und möglicherweise ein Befall myokardialer Zellen entscheidend. In Fällen mit schwerer Anämie oder ausgeprägtem Hydrops konnte die fetale Mortalität durch intrauterine Transfusionen gesenkt werden. Postnatal ist die adaptive humorale Immunantwort für die Bekämpfung der
B19V-Infektionen entscheidend. Hingegen liegen nur wenige Erkenntnisse über die fetale Immunantwort gegen B19V vor. Bei immundefizienten Patienten erwies sich die Gabe von intravenösem Immunglobulin als effektiv. Auch gibt es Fallberichte über die Injektion von Immunglobulin bei hydropischen Feten direkt in das fetale Peritoneum, die zu einer klinischen Besserung führten. In dieser retrospektiven Studie aus Deutschland wurde das virologische Muster im mütterlichen und fetalen Blut bei 41 Fällen einer fetalen B19V-Infektion untersucht. Zum Zeitpunkt der Blutabnahme beim Feten waren die Mütter alle B19V-DNA sowie Anti-B19V-IgG positiv, bei 95\% ließ sich Anti-B19V-IgM nachweisen. Im Fetalblut wurde in 100\% B19V-DNA, in 28\% Anti-B19V-IgM und in 24\% Anti-B19V-IgG nachgewiesen. Die Wahrscheinlichkeit des Vorliegens von B19V-spezifischem IgM- und IgG-Anti- körpern stieg mit zunehmendem Gestationsalter an. Es zeigte sich ferner, dass die Anti-B19V-Konzentration der Mutter nicht mit der Nachweiswahrscheinlichkeit beim Feten korrelierte. Hingegen fand sich ein Zusammenhang zwischen dem Vorhandensein von fetalen Anti-B19V-IgG und einer niedrigen B19V-Viruslast bei der Mutter sowie einem weniger schweren klinischen Bild beim Feten.

Fazit: Die Studie trägt zum besseren Verständnis zur Ätiologie und zum Verlauf einer fetalen B19V-Infektion bei. Ein Fehlen oder ein eingeschränkter maternofetaler Transfer von mütterlichen IgGAntikörpern erklären infolge der fehlenden Virusneutralisation das Auftreten der fetalen Anämie sowie des Hydrops. Auffällig war auch das Ausbleiben einer messbaren fetalen IgM-Antwort in 75\% der Fälle. Als Konsequenz bedeutet dies, dass der Nachweis von fetalem AntiB19V-IgM nur im positiven Fall eine Aussagekraft hat. Für den sicheren Nachweis einer fetalen B19V-Infektion ist daher nur der B19V-DNA-Nachweis mittels PCR relevant.

Prof. Tino F. Schwarz

Weiffenbach J et al.: Serological and virological analysis of maternal and fetal blood samples in prenatal human parvovirus B19 infection. J. Infect. Dis. 2012; 205:782-8

\section{Plazenta lösen: Zug an der Nabelschnur sinnvoll?}

\section{Zur Prävention postpartaler Blutungen wird in vielen Entwicklungsländern die Cord-Traction eingesetzt. Gibt es mit Oxytocin weniger blutungsbedingte Todesfälle von Frauen im Wochenbett?}

U $\mathrm{m}$ das Risiko schwerer postpartaler Blutungen zu senken, erfolgt in vielen Ländern in der Nachgeburtsphase ein sogenanntes „aktives Management": neben dem Abklemmen der Nabelschnur und der Oxytocin-Gabe auch der kontrollierte Zug an der Nabelschnur (CordTraction), um die Plazenta zu lösen. Dass auf die häufig eingesetzte Cord-Traction, die einige geburtshilfliche Erfahrung voraussetzt und zudem mit dem Risiko der Uterusinversion verbunden ist, verzichtet werden kann, zeigte kürzlich eine von der WHO und Weltbank geförderte Studie. Rund 25.000 Gebärende aus acht
Entwicklungsländern nahmen teil. In Gruppe 1 erhielten die Frauen unmittelbar nach der Entbindung 10 IU Oxytocin i.m.; innerhalb von einer halben Stunde nach Geburt erfolgte die Cord-Traction. Frauen aus Gruppe 2 erhielten auch Oxytocin, jedoch vorerst keine Cord-Traction. Bei $6 \%$ von ihnen war die Cord-Traction letztlich doch erforderlich, weil die Plazenta sich nicht von selbst gelöst hatte.

Das Risiko schwerer Blutverluste von 1 l oder mehr war in beiden Gruppen vergleichbar. Zwar wurde diese kritische Marke in der Gruppe ohne Cord-Traction häufiger überschritten, sodass sich die
Nichtunterlegenheit des Verzichts auf den Nabelschnurzug im primären Studienendpunkt nicht zeigen ließ. Dafür betrug die „number needed to harm“ 581 , das heißt auf 581 Frauen, die keine CordTraction erhielten, kam eine Gebärende mit schwerer postpartaler Blutung.

Fazit: Zur postpartalen Blutungsprävention ist die uterotonische Komponente mit Oxytocin wichtig. Außerklinische Einrichtungen sollten sich darauf konzentrieren, v.a. wenn kein in der Cord-Traction erfahrener Geburtshelfer anwesend ist.

Dr. Elke Oberhofer

Gülmezoglu AM et al. Active management of the third stage of labour with and without controlled cord traction: a randomised, controlled, non-inferiority trial. Lancet 2012; DOI:10.1016/S0140-6736(12)60206-2 